\title{
Home-Based Pulmonary Rehabilitation for Subjects With COPD: A Randomized Study
}

\author{
Cristiane O Pradella PT MSc, Graziela M Belmonte PT, Marcilene N Maia PT MSc, \\ Cionéia S Delgado PT MSc, Ana Paula T Luise PT MSc, Oliver A Nascimento MD, \\ Mariana R Gazzotti PhD, and José R Jardim MD
}

\begin{abstract}
BACKGROUND: The aim of this study was to develop an efficient, low-cost, home-based pulmonary rehabilitation program and to evaluate the impact of the program on exercise as measured by the 6-min walk test (6MWT) and treadmill endurance test. METHODS: Twenty-nine subjects with $\operatorname{COPD}\left(\mathrm{FEV}_{1}=62.4 \pm 10.7 \%\right.$ of predicted, $62.4 \pm 10.7 \mathrm{y}$ old $)$ were included in a randomized and prospective pulmonary rehabilitation program, and they performed 24 sessions, $5 \mathrm{~d} /$ week. The control group included 15 subjects $\left(\mathrm{FEV}_{1}=54 \pm 26.2 \%\right.$ of predicted, $65.3 \pm 8 \mathrm{y}$ old $)$. They were evaluated pre-intervention and post-intervention by the 6MWT, St George Respiratory Questionnaire (SGRQ), treadmill endurance test, and spirometry. The home-based pulmonary rehabilitation program consisted of walking for $\mathbf{4 0}$ min along a corridor or a street, climbing stairs for $\mathbf{1 5} \mathrm{min}$, and exercising the arms with an oil can $(1 \mathrm{~kg})$ using diagonal movements for $15 \mathrm{~min}$. Subjects were called once each week for encouragement and verification of adherence. Both groups received the usual pharmacologic treatment; in addition, the control group received a telephone call without guidance on exercise. RESULTS: The 2 groups were similar regarding age, $\mathrm{FEV}_{1}$, and FVC. The home-based pulmonary rehabilitation group increased by $65 \mathrm{~m}$ in the 6MWT $(P<.05)$ and $316.6 \pm 81.8 \mathrm{~m}$ in the endurance test $(P<.05)$ and decreased by $>4$ units in all SGRQ domains. The control group showed no difference in any variable. CONCLUSIONS: This study demonstrates that a simple, low-cost, home-based pulmonary rehabilitation program adapted to real-life situations leads to improvement in exercise capacity and quality of life. Key words: chronic obstructive pulmonary disease; home pulmonary rehabilitation; pulmonary rehabilitation; exercise capacity; 6MWT; COPD. [Respir Care 2015;60(4):526-532. (C) 2015 Daedalus Enterprises]
\end{abstract}

\section{Introduction}

Pulmonary rehabilitation is defined as a multidisciplinary health-care program for patients with chronic respiratory

\footnotetext{
Ms Pradella, Ms Belmonte, Ms Maia, Ms Delgado, Ms Luise, and Drs Nascimento, Gazzotti, and Jardim are affiliated with the Pulmonary Rehabilitation Center, and Drs Nascimento and Jardim are also affiliated with the Respiratory Division, Escola Paulista de Medicina, Universidade Federal de São Paulo, São Paulo, Brazil.
}

The authors have disclosed no conflicts of interest.

Correspondence: José R Jardim MD, Respiratory Division, Escola Paulista de Medicina, Rua Botucatu 740, $3^{\circ}$ Anda, 04023-062 São Paulo, SP, Brazil. E-mail: jardimpneumo@gmail.com.

DOI: $10.4187 /$ respcare.02994 conditions, aimed at optimizing autonomy in terms of physical and social performance. The goal of pulmonary rehabilitation in such cases is to offer individualized treatment to reduce symptoms, optimize functional capacity, enhance social participation, and reduce health-care costs by stabilizing or reducing the systemic manifestations of the disease. ${ }^{1-3}$

The current non-drug treatment of COPD is based on rehabilitation involving oxygen therapy, psychological treatment, nutrition, and physical exercise. ${ }^{2}$ The latter component is essential and results in greater tolerance to exercise, decrease in dyspnea, and improved quality of life. ${ }^{2,3}$

Because of the limited number of rehabilitation centers to meet the needs of all patients with COPD, home-based pulmonary rehabilitation programs have been proposed. However, studies assessing such programs have had drawbacks, such as small numbers of subjects, ${ }^{4,5}$ initiation of 


\section{Home-Based Pulmonary Rehab for Subjects With COPD}

the program during exacerbation, ${ }^{6}$ combination of the program with respiratory physical therapy, ${ }^{7}$ and involvement of only subjects with very severe COPD. ${ }^{8}$ Maltais et $\mathrm{al}^{9}$ carried out the most important investigation of homebased pulmonary rehabilitation programs in a randomized study involving an adequate number of subjects and assessing subject response in terms of dyspnea. They found that a home-based pulmonary rehabilitation program with a cycle ergometer was as efficacious for patients with COPD as rehabilitation in a hospital setting. ${ }^{9}$ However, training with a cycle ergometer implies costs to the patient, which may be an obstacle to the widespread use of this protocol.

Besides offering the same physiological benefits as exercise performed in a hospital setting, a home-based pulmonary rehabilitation program can be inexpensive and easy to perform. In addition, the patient is kept close to the family, which might help motivate the patient to reestablish independence in terms of daily activities.

The hypothesis was that patients with COPD trained in a home-based pulmonary rehabilitation program achieve better physical capacity than patients without such training. The aims of this study were (1) to develop an efficient, low-cost, home-based pulmonary rehabilitation program; (2) to evaluate the impact of the program on exercise as measured by the 6-min walk test (6MWT) and treadmill endurance test; and (3) to assess the effect on quality of life in a group of subjects with COPD under partial supervision compared with a control group.

\section{Methods}

The study protocol was approved by the Ethics Committee on Research of the Federal University of São Paulo (CEP 0677/05), and the subjects provided signed informed consent. Fifty subjects were recruited for the study, with the following inclusion criteria: diagnosis of COPD based on the criteria of the Brazilian Thoracic Society and Global Initiative for Chronic Obstructive Lung Disease (GOLD), , $\mathrm{FEV}_{1}<80 \%$ of predicted, between 40 and $75 \mathrm{y}$ of age, either gender, and signed informed consent. Exclusion criteria included participation in a pulmonary rehabilitation program in the previous 12 months; regular practice of any type of physical activity, such as fast walking, swimming, or exercise at a fitness center for at least $40 \mathrm{~min}$, 3 times/week, in the previous 12 months; presence of other disease that could lead to exercise intolerance, such as neurological, heart, or orthopedic conditions; cognitive impairment; and exacerbation in the 4 weeks before the study. All subjects were evaluated before the intervention by spirometry, 6MWT, and a lower limb endurance test; they also completed the St George Respiratory Questionnaire (SGRQ) to assess quality of life. The subjects underwent the same evaluation procedures after 24 training sessions.

\section{QUICK LOOK}

\section{Current knowledge}

Pulmonary rehabilitation is defined as a multidisciplinary program for patients with chronic respiratory conditions, aimed at optimizing autonomy in terms of physical and social performance. The aim of pulmonary rehabilitation is to offer individualized treatment to reduce symptoms, optimize functional capacity, enhance social participation, and reduce health-care costs by stabilizing or reducing the systemic manifestations of the disease.

\section{What this paper contributes to our knowledge}

A simple, low-cost, home-based pulmonary rehabilitation program adapted to real-life situations leads to improvements in exercise capacity, shortness of breath, and quality of life. These findings underscore the importance of home-based pulmonary rehabilitation as a treatment option for patients with COPD. Further studies should evaluate long-term adherence.

\section{Protocol}

Following the initial evaluation, 50 subjects were randomly allocated to either a home-based rehabilitation group (32 subjects), with a home-based pulmonary rehabilitation program, or a control group (18 subjects), with no intervention. The subjects in the home-based rehabilitation group spent a week at the rehabilitation center learning the exercises and received printed material (educational booklet) describing the exercises to be performed (warm-up, aerobic activity, stretching, and relaxation), including frequency (3 times/week) and duration of the program (24 sessions). Subjects also received a $\log$ to record their activities. Both groups received a weekly telephone call from a trained professional, during which the subjects were asked about their clinical status, and those in the homebased rehabilitation group were encouraged to adhere to the program.

Three subjects in the control group discontinued the study: 2 withdrew their consent, and one experienced a severe exacerbation. In the home-based rehabilitation group, one patient died, one withdrew his consent, and one experienced an exacerbation requiring hospitalization. Thus, the final control sample included 15 subjects, and the study group included 29 subjects. All subjects in both groups maintained their usual medications throughout the study. 


\section{Home-Based Pulmonary Rehab for Subjects With COPD}

\section{Evaluations}

Pre-bronchodilator and post-bronchodilator (400 $32 \mu \mathrm{g}$ of salbutamol) spirometries were performed using a portable spirometer (EasyOne, ndd Medical Technologies, Chelmsford, Massachusetts) ${ }^{10}$ that was checked daily with a 3-L calibration syringe. At least 3 measures were taken following the criteria of reproducibility and acceptability recommended by the American Thoracic Society. The predicted values were calculated based on the third National Health and Nutrition Examination Survey. ${ }^{11}$

The SGRQ version used in this study was validated for use in Brazil by Sousa et al. ${ }^{12}$ The total and domain scores range from 0 to 100 , with higher scores denoting poorer quality of life. A minimum difference of 4 units is considered clinically important. ${ }^{12}$

The 6MWT was performed in a corridor with a flat surface, with the subject receiving verbal encouragement at 1-min intervals. The following variables were measured before and after each test: heart rate, breathing frequency, blood pressure, hemoglobin saturation $\left(\mathrm{S}_{\mathrm{pO}_{2}}\right)$, and dyspnea and lower limb fatigue (using the Borg scale). If excessive fatigue was experienced, the subject was allowed to stop without stopping the chronometer. The test was performed twice during each evaluation (pre-intervention and postintervention). ${ }^{13}$

The lower limb maximum incremental test was performed on a treadmill following the Harbor protocol. The subject began at a comfortable pace, and the inclination of the treadmill was increased by $1 \%$ at $1-$ min intervals. The test was symptom-limited and interrupted when the subject could no longer continue; when 15 min had passed; or if the subject experienced dizziness, nausea, vomiting, paleness, blood pressure $>220 / 120 \mathrm{~mm} \mathrm{Hg}$, electrocardiogram alterations, or intense precordial pain. The aim of the test was to determine the load to use during the endurance test. ${ }^{2}$ Electrocardiogram, heart rate, breathing frequency, blood pressure, $\mathrm{S}_{\mathrm{pO}_{2}}$, and Borg scales for dyspnea and lower limb fatigue were determined at rest, after a 3-min of warm-up, and every 3 min during the test until the end of the test.

The lower limb endurance test was performed on a treadmill at the same speed and at $90 \%$ of the maximum inclination reached on the incremental test. The same variables mentioned above were determined at rest and every $3 \mathrm{~min}$ during the test until the end of the test. The test was interrupted using the same criteria as for the incremental test. At the end of the test, the time and distance walked by the subject were recorded. ${ }^{3}$

\section{Rehabilitation Program}

The home-based pulmonary rehabilitation program was composed of 4 steps: (1) warm-up, (2) aerobic activ- ity, (3) stretching, and (4) relaxation. In step 1, the warm-up included five 1-min exercises with a 1-min rest interval between exercises. In step 2, the aerobic activity was divided into 3 stages, with a progressive increase in the degree of difficulty and number of activities. In stage I (first week), the subjects walked at home if they had a corridor at least $25 \mathrm{~m}$ long; if not, they were instructed to walk on the street or in a park for $40 \mathrm{~min}$ (at a slow pace for the first $5 \mathrm{~min}$, an increased pace for the next $30 \mathrm{~min}$, and a slow pace for the last $5 \mathrm{~min}$ ), with a heart rate of $60-70 \%$ of the maximum heart rate for the subject's age (maximum heart rate $=220-$ age). Subjects unable to walk for $40 \mathrm{~min}$ during the first session were instructed to walk for 15 min during the first week and to increase the time gradually. In stage II (second week), the subjects walked in the morning and went up and down stairs in the afternoon. The stair exercise began with $5 \mathrm{~min} / \mathrm{d}$, increasing to 15 min after 2 weeks. If a subject did not have stairs at home, a platform $20 \mathrm{~cm}$ in height and $50 \mathrm{~cm}$ in depth was provided so that the subject could simulate going up and down a step for the established time. In stage III (second week), in addition to the walking and stair exercises, the subjects performed upper limb exercises, which consisted of diagonal movements with a $1-\mathrm{kg}$ load (oil can). The subjects performed 3 sets of 30 movements per arm, with 1-min rest intervals between sets. Step 3 consisted of stretching after the exercise sessions, with the goal of diminishing muscle tension and pain; muscle groups of the head and neck region and of the upper and lower limbs were stretched for $\sim 20 \mathrm{~s} /$ position. ${ }^{2}$ In step 4 (relaxation), the subjects remained in a reclining position with their heads on a pillow, listening to music at a comfortable volume. The subjects breathed deeply 3 times, closed their eyes to feel their bodies better in time and space, and concentrated on the parts of the body in contact with the floor or chair. The relaxation exercise lasted $\sim 30 \mathrm{~min}$.

On rainy days, the subjects performed the stair exercise in both the morning and afternoon. The subjects were instructed to use pursed-lip breathing during the walking and stair exercises. Subjects were required to continue their regular medications and stair exercises throughout the study.

\section{Statistical Analysis}

Data were expressed as mean \pm SD and $95 \%$ CI. Paired $t$ tests were used to compare the pre-intervention and postintervention data to determine possible intragroup differences. Unpaired $t$ tests were used to determine possible differences between the home-based rehabilitation and control groups. The level of significance was set to $5 \%$ $(P<.05)$. Analyses were performed with SPSS 16.0 (SPSS, Chicago, Illinois). 


\section{Home-Based Pulmonary Rehab for Subjects With COPD}

As pulmonary rehabilitation is a mandatory part of the treatment, we considered that it would not be ethical to have a large control group and not allow subjects to take advantage of the program. Fifty subjects on the rehabilitation waiting list were selected, and after the initial evaluation, they were randomized at a proportion of 1:1.5 to the home-based rehabilitation or control group. We calculated the sample size with $\mathrm{G}^{*}$ Power software, ${ }^{14}$ using data based on a previous home-based pulmonary rehabilitation study. ${ }^{15}$ That study reported that the home-based pulmonary rehabilitation group had an improvement of $79 \mathrm{~m}$ in 6-min walk distance over the control group, with an SD of $82 \mathrm{~m}$ (effect size of 0.96). Assuming an $\alpha$ of 0.05 and a $\beta$ of 0.80 , the sample size needed was 38 subjects: 23 in the home-based rehabilitation group and 15 in the control group.

\section{Results}

Table 1 displays the demographic data of the 44 subjects. Most of the subjects were male, and no significant differences between groups were found regarding age, $\mathrm{FEV}_{1}$, or FVC. Table 2 displays the 6MWT results: the home-based rehabilitation group walked a significantly longer distance, with a mean increase of $65.7 \pm 83.1 \mathrm{~m}$ $(P=.039)$, whereas the control group walked only an additional $5.5 \pm 92.9 \mathrm{~m}(P=.34)$. Table 3 displays the results of the endurance test: the home-based rehabilitation group demonstrated a significant increase $(316.6 \pm 81.8 \mathrm{~m})$ in distance walked $(P=.001)$, whereas the control group walked an additional $31.2 \pm 419.7 \mathrm{~m}(P=.66)$.

Table 4 displays the results of the SGRQ. The homebased rehabilitation group reached statistically and clinically important reductions in all domain scores, whereas no significant differences were found in the control group.

\section{Discussion}

This study demonstrates that a low-cost, home-based pulmonary rehabilitation program is effective and improves shortness of breath, tolerance to exercise, and quality of life in patients with COPD. These findings lend support to the hypothesis that home-based pulmonary rehabilitation can enhance the physical capacity of patients with COPD and reduce their symptoms.

Physical training is the basis of pulmonary rehabilitation and has been shown to enhance performance; promote physiological adaptations in peripheral muscles; and improve the sensation of shortness of breath, heart function, emotional state, and quality of life. ${ }^{2}$ At the end of the home-based pulmonary rehabilitation program in this study, the subjects showed a mean increase in walk distance of $\sim 65 \mathrm{~m}$, compared with $6 \mathrm{~m}$ in the control group. Studies have concluded that $25 \mathrm{~m}^{16}$ to $30 \mathrm{~m}^{17}$ is the min-
Table 1. Characteristics of Subjects in Control and Home-Based Rehabilitation Groups

\begin{tabular}{|c|c|c|}
\hline Variable* & $\begin{array}{l}\text { Control Group } \\
\quad(n=15)\end{array}$ & $\begin{array}{c}\text { Home-based } \\
\text { Rehabilitation } \\
\text { Group } \\
(n=29)\end{array}$ \\
\hline Age (mean $\pm S D), y$ & $65.3 \pm 8$ & $62.4 \pm 10.7$ \\
\hline \multicolumn{3}{|l|}{ Gender, $n(\%)$} \\
\hline Female & $2(13.3)$ & $6(20.7)$ \\
\hline Male & $13(86.7)$ & $23(79.3)$ \\
\hline \multicolumn{3}{|l|}{ GOLD stage, $n(\%)$} \\
\hline Mild & $1(6.7)$ & $1(3.4)$ \\
\hline Moderate & $8(53.3)$ & $6(20.7)$ \\
\hline Severe & $3(20.0)$ & $14(48.3)$ \\
\hline Very severe & $3(20.0)$ & $3(27.6)$ \\
\hline $\mathrm{BMI}($ mean $\pm \mathrm{SD}), \mathrm{kg} / \mathrm{m}^{2}$ & $26.7 \pm 5.3$ & $25.2 \pm 5.0$ \\
\hline $\mathrm{FEV}_{1}($ mean $\pm \mathrm{SD}), \mathrm{L}$ & $1.5 \pm 0.6$ & $1.2 \pm 0.4$ \\
\hline $\mathrm{FEV}_{1}($ mean $\pm \mathrm{SD}), \%$ predicted & $54.0 \pm 22.2$ & $43.9 \pm 16.2$ \\
\hline $\mathrm{FVC}$ (mean $\pm \mathrm{SD}), \mathrm{L}$ & $3.1 \pm 0.7$ & $2.8 \pm 0.8$ \\
\hline FVC $($ mean $\pm \mathrm{SD}), \%$ predicted & $81.1 \pm 17.9$ & $74.3 \pm 15.1$ \\
\hline $\mathrm{FEV}_{1} / \mathrm{FVC}($ mean $\pm \mathrm{SD})$ & $0.48 \pm 0.14$ & $0.45 \pm 0.13$ \\
\hline \multicolumn{3}{|c|}{$\begin{array}{l}\text { * Non-significant differences for all variables } \\
\text { GOLD = Global Initiative for Chronic Obstructive Lung Disease } \\
\text { BMI = body mass index }\end{array}$} \\
\hline
\end{tabular}

imum clinically important difference in this test. Changes in absolute values are considered to be more sensitive indicators than changes in percentage values. ${ }^{18}$ The improvement seen in our study suggests that a home-based pulmonary rehabilitation program can be recommended as an alternative to conventional pulmonary rehabilitation carried out in a hospital setting to enhance tolerance to exercise in patients with COPD. To the best of our knowledge, this is the first study to demonstrate that a simple, low-cost protocol is effective for this purpose. Likewise, the greater walk distance on the endurance test further demonstrates the improvement these subjects achieved with the home-based pulmonary rehabilitation program (see Table 3).

In a well-designed study, Maltais et $\mathrm{al}^{9}$ demonstrated that a home-based pulmonary rehabilitation program is as effective as conventional pulmonary rehabilitation in patients with moderate-to-severe COPD. After 8 weeks of training, subjects achieved a better performance and a reduction in shortness of breath, which lasted up to $1 \mathrm{y}$ after the intervention. However, the protocol involved the use of a cycle ergometer, which is economically unfeasible in most countries.

The simple, low-cost, and easy-to-execute protocol described in this study seems to be adequate for the majority of patients with COPD. An attempt was made to stimulate adherence through weekly telephone contact to encourage the subjects to perform the exercises. The fact that only one subject in the home-based rehabilitation group withdrew from the study demonstrates that partial supervision 


\section{Home-Based Pulmonary Rehab for Subjects With COPD}

Table 2. Variables Before and After the 6-Min Walk Test in Control and Home-Based Rehabilitation Groups

\begin{tabular}{|c|c|c|c|c|c|c|c|}
\hline \multirow[b]{2}{*}{ Variable } & \multicolumn{3}{|c|}{ Control Group $(n=15)$} & \multicolumn{3}{|c|}{ Home-Based Rehabilitation Group $(n=29)$} & \multirow{2}{*}{$\begin{array}{l}\text { Home-Based } \\
\text { Rehabilitation vs Control } \\
\quad(95 \% \mathrm{CI})\end{array}$} \\
\hline & Initial & Final & $\begin{array}{c}\text { Difference } \\
\text { (Final - Initial) }\end{array}$ & Initial & Final & $\begin{array}{c}\text { Difference } \\
\text { (Final - Initial) }\end{array}$ & \\
\hline Distance, $\mathrm{m}$ & $456.5 \pm 71.1$ & $462.1 \pm 101.4$ & $5.5 \pm 92.9$ & $485.1 \pm 79.6$ & $550.8 \pm 100.7$ & $65.7 \pm 83.1^{*}$ & $60.2(4.6-115.7)^{*}$ \\
\hline \multicolumn{8}{|c|}{ Heart rate, beats/min } \\
\hline Initial & $84.6 \pm 12.4$ & $84.6 \pm 13.6$ & $0 \pm 11.4$ & $87.3 \pm 12.3$ & $89.9 \pm 13.7^{*}$ & $2.6 \pm 8.8$ & $2.6(-3.6$ to 8.9$)$ \\
\hline Final & $106.1 \pm 17$ & $109.4 \pm 17.9$ & $3.3 \pm 13.2$ & $117.1 \pm 16$ & $118.2 \pm 12.9$ & $1.1 \pm 12.0$ & $2.2(-10.1$ to 5.8$)$ \\
\hline \multicolumn{8}{|c|}{$\begin{array}{c}\text { Breathing frequency, } \\
\text { breaths/min }\end{array}$} \\
\hline Initial & $18.5 \pm 3.6$ & $18.1 \pm 5$ & $-0.4 \pm 6.2$ & $19.5 \pm 3.5$ & $18.4 \pm 3.4$ & $-1.0 \pm 3.8$ & $0.6(-3.7$ to 2.4$)$ \\
\hline Final & $26.2 \pm 7.2$ & $26 \pm 5.9$ & $-0.3 \pm 4.1$ & $27.2 \pm 4.3$ & $26 \pm 4.1$ & $-3.4 \pm 5.1$ & $0.1(-3.2$ to 2.9$)$ \\
\hline \multicolumn{8}{|l|}{$\mathrm{SBP}, \mathrm{mm} \mathrm{Hg}$} \\
\hline Initial & $118.6 \pm 11.2$ & $127 \pm 17.5$ & $8.7 \pm 21.0$ & $126.5 \pm 13.9$ & $124 \pm 10.2$ & $-2.1 \pm 14.0$ & $10.7(-21.4 \text { to }-0.0)^{*}$ \\
\hline Final & $138.6 \pm 16.8$ & $148.6 \pm 18.1$ & $10.0 \pm 19.7$ & $157.6 \pm 23.8$ & $153.1 \pm 21.4$ & $-4.4 \pm 23.2^{*}$ & $14.5(-28.7 \text { to }-0.2)^{*}$ \\
\hline \multicolumn{8}{|l|}{$\mathrm{DBP}, \mathrm{mm} \mathrm{Hg}$} \\
\hline Initial & $82.0 \pm 7.7$ & $79.3 \pm 11.6$ & $-2.7 \pm 13.9$ & $81.7 \pm 12.5$ & $75.5 \pm 12.4$ & $-6.2 \pm 14.5$ & $3.5(-12.7$ to 5.6$)$ \\
\hline Final & $84.6 \pm 8.3$ & $82.6 \pm 11.6$ & $-2.0 \pm 12.7$ & $87.2 \pm 11.3$ & $85.8 \pm 12.7$ & $-1.4 \pm 15.7$ & $-0.6(-8.9$ to 10.1$)$ \\
\hline \multicolumn{8}{|l|}{$\begin{array}{c}\text { Borg scale for } \\
\text { dyspnea }\end{array}$} \\
\hline Initial & $0.4 \pm 0.7$ & $0.6 \pm 1.3$ & $0.2 \pm 1.3$ & $0.4 \pm 0.7$ & $0.3 \pm 0.8$ & $-0.1 \pm 0.6$ & $0.36(-0.9$ to 0.2$)$ \\
\hline Final & $2.2 \pm 2.4$ & $2.7 \pm 2.5$ & $0.5 \pm 1.7$ & $3.3 \pm 2.7$ & $3.1 \pm 2.2$ & $-0.2 \pm 3.0$ & $0.7(-2.4$ to 1.0$)$ \\
\hline \multicolumn{8}{|c|}{$\begin{array}{l}\text { Borg scale for leg } \\
\text { fatigue }\end{array}$} \\
\hline Initial & $0.6 \pm 1$ & $0.5 \pm 1.1$ & $-0.3 \pm 1.5$ & $0.4 \pm 0.7$ & $0.3 \pm 0.6$ & $-0.1 \pm 0.8$ & $0.1(-0.8$ to 0.6$)$ \\
\hline Final & $2.2 \pm 2.4$ & $2.5 \pm 2.3$ & $0.3 \pm 1.7$ & $2.8 \pm 2.7$ & $2.3 \pm 1.8$ & $-0.4 \pm 2.8$ & $0.7(-2.2$ to 0.9$)$ \\
\hline \multicolumn{8}{|l|}{$\mathrm{S}_{\mathrm{pO}_{2}}, \%$} \\
\hline Initial & $93.8 \pm 2$ & $94.0 \pm 2$ & $0.0 \pm 0.0$ & $93.3 \pm 2$ & $93 \pm 2$ & $0.0 \pm 0.0$ & $0(0.0-0.0)$ \\
\hline Final & $90.1 \pm 4$ & $90.1 \pm 4$ & $0.0 \pm 0.0$ & $88.2 \pm 4$ & $88.2 \pm 4$ & $0.0 \pm 0.0$ & $0(0.0-0.0)$ \\
\hline \multicolumn{8}{|c|}{ 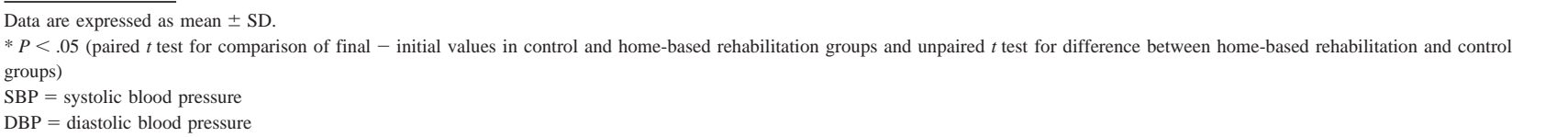 } \\
\hline
\end{tabular}

can help in maintaining adherence to a proposed exercise program on the part of subjects with COPD. There is evidence that patients with chronic diseases may exhibit low adherence to treatment. ${ }^{19,20}$

Dyspnea is an important limiting factor for exertion in patients with COPD. Thus, it is to be expected that any intervention that helps to diminish dyspnea should be associated with improved functional capacity and quality of life in patients with COPD. In this study, only the subjects in the home-based rehabilitation group exhibited improvement in the symptom domain of the SGRQ, which is related to dyspnea. As shortness of breath limits physical activities, the clinically important 2 -fold reduction in the activity domain score may also be considered as an indirect measure of improvement in dyspnea (see Table 4). Despite using different protocols, other studies on homebased rehabilitation have also demonstrated that dyspnea may be reduced in patients with COPD. 4,9,21,22
Different factors might have influenced improvements in quality of life. The subjects also learned to use their medications correctly, which may have contributed to improved respiratory function and a possible reduction in pulmonary hyperinflation, ${ }^{18,23,24}$ resulting in improved physical capacity. ${ }^{2}$ Moreover, the weekly telephone calls might have made the subjects feel more protected and encouraged. Thus, the improvement in quality of life likely occurred from a combination of these factors, rather than merely the training itself.

This study has limitations that should be addressed. First, there was no long-term follow-up of the subjects in the home-based rehabilitation group, and we do not know how long these subjects continued the exercises on their own. However, all of the subjects were instructed on the benefits of performing regular physical activity for the rest of their lives. Despite this limitation, this is the first randomized controlled clinical trial with a simple, low-cost pul- 


\section{Home-Based Pulmonary Rehab for Subjects With COPD}

Table 3. Variables Before and After the Endurance Test in the Control and Home-Based Rehabilitation Groups

\begin{tabular}{|c|c|c|c|c|c|c|c|}
\hline \multirow[b]{2}{*}{ Variable } & \multicolumn{3}{|c|}{ Control Group $(n=15)$} & \multicolumn{3}{|c|}{ Home-Based Rehabilitation Group $(n=29)$} & \multirow{2}{*}{$\begin{array}{c}\text { Home-Based } \\
\text { Rehabilitation vs Control } \\
(95 \% \mathrm{CI})\end{array}$} \\
\hline & Initial & Final & $\begin{array}{c}\text { Difference } \\
\text { (Final - Initial) }\end{array}$ & Initial & Final & $\begin{array}{c}\text { Difference } \\
\text { (Final - Initial) }\end{array}$ & \\
\hline Distance, $\mathrm{m}$ & $923.7 \pm 588.8$ & $954.9 \pm 572.4$ & $31.2 \pm 419.7$ & $708.4 \pm 364.4$ & $1,025.0 \pm 706.2$ & $316.6 \pm 81.8^{*}$ & $285.42(-7.1 \text { to } 563.8)^{*}$ \\
\hline Inclination, $\%$ & $9.6 \pm 2.0$ & $9.4 \pm 1.9$ & $0.0 \pm 0.0$ & $9.7 \pm 1.2$ & $9.7 \pm 1.2$ & $0.0 \pm 0.0$ & $0.0(0.0-0.0)$ \\
\hline Speed, miles/h & $2.0 \pm 0.6$ & $2.0 \pm 0.6$ & & & & & \\
\hline Time, s & $969.7 \pm 500.6$ & $1,052.6 \pm 661.8$ & $82.8 \pm 479.1$ & $698.1 \pm 364.0$ & $1,045 \pm 765.8$ & $347.6 \pm 87.4 \dagger$ & $264.8(-46.3$ to 575.8$) \dagger$ \\
\hline \multicolumn{8}{|c|}{ Heart rate, beats/min } \\
\hline Initial & $82.2 \pm 13.5$ & $82.20 \pm 14.9$ & $-0.1 \pm 19.4$ & $89.4 \pm 20.0$ & $86.9 \pm 11.1$ & $-2.5 \pm 20.4$ & $2.5(-15.3$ to 10.4$)$ \\
\hline Final & $123.0 \pm 17.8$ & $117.5 \pm 37.2$ & $-5.5 \pm 35.1$ & $136.6 \pm 14.6$ & $136.2 \pm 16.5$ & $-0.4 \pm 15.5$ & $-5.1(-10.2$ to 20.5$)$ \\
\hline \multicolumn{8}{|c|}{$\begin{array}{l}\text { Breathing frequency, } \\
\text { breaths/min }\end{array}$} \\
\hline Initial & $17.9 \pm 3.7$ & $18.8 \pm 4.0$ & $0.8 \pm 5.5$ & $17.7 \pm 5.0$ & $17.7 \pm 4.0$ & $0.0 \pm 6.8$ & $0.8(-4.9$ to 3.3$)$ \\
\hline Final & $32.2 \pm 3.0$ & $31.5 \pm 6.1$ & $-0.8 \pm 5.7$ & $32.7 \pm 5.0$ & $31.5 \pm 4.5$ & $-1.2 \pm 5.5$ & $0.4(-4.0$ to 3.2$)$ \\
\hline \multicolumn{8}{|l|}{$\mathrm{SBP}, \mathrm{mm} \mathrm{Hg}$} \\
\hline Initia & $123.3 \pm 11.7$ & $121.3 \pm 14.0$ & $-2.0 \pm 14.2$ & $122.4 \pm 13.2$ & $124.1 \pm 9.1$ & $1.7 \pm 16.5$ & $-3.7(-6.4$ to 13.8$)$ \\
\hline Final & $158.0 \pm 24.8$ & $152.0 \pm 24.5$ & $-6.0 \pm 23.2$ & $167.9 \pm 22.7$ & $157.2 \pm 19.6$ & $-10.6 \pm 25.3$ & $4.7(-20.5$ to 11.1$)$ \\
\hline \multicolumn{8}{|l|}{$\mathrm{DBP}, \mathrm{mm} \mathrm{Hg}$} \\
\hline Initial & $80.0 \pm 9.2$ & $78.0 \pm 9.4$ & $-2.0 \pm 10.8$ & $75.8 \pm 9.8$ & $76.5 \pm 8.1$ & $0.7 \pm 11.3$ & $-2.7(-4.4$ to 9.8$)$ \\
\hline Final & $92.6 \pm 13.3$ & $89.3 \pm 10.3$ & $-3.3 \pm 12.9$ & $91.0 \pm 11.7$ & $90.0 \pm 11.9$ & $-1.0 \pm 9.4$ & $-2.3(-4.5$ to 9.1$)$ \\
\hline \multicolumn{8}{|l|}{$\begin{array}{c}\text { Borg scale for } \\
\text { dyspnea }\end{array}$} \\
\hline Initial & $0.26 \pm 0.8$ & $0.33 \pm 0.7$ & $0.0 \pm 0.6$ & $0.24 \pm 0.6$ & $0.13 \pm 0.4$ & $-0.1 \pm 0.3$ & $0.2(-0.4$ to 0.1$)$ \\
\hline Final & $6.8 \pm 2.8$ & $7.0 \pm 2.8$ & $0.1 \pm 2.4$ & $6.8 \pm 3.4$ & $6.6 \pm 3.0$ & $-0.2 \pm 2.8$ & $0.3(-2.1$ to 1.4$)$ \\
\hline \multicolumn{8}{|c|}{$\begin{array}{l}\text { Borg scale for leg } \\
\text { fatigue }\end{array}$} \\
\hline Initial & $0.33 \pm 0.8$ & $0,5 \pm 0,9$ & $0.1 \pm 1.1$ & $0.32 \pm 0.7$ & $0.2 \pm 0.4$ & $-0.1 \pm 0.5$ & $0.3(-0.8$ to 0.2$)$ \\
\hline Final & $6.6 \pm 3.0$ & $6.4 \pm 3.4$ & $-0.1 \pm 3.2$ & $6.0 \pm 3.2$ & $6.7 \pm 2.8$ & $0.7 \pm 2.6$ & -0.8 ( -0.9 to 2.7$)$ \\
\hline \multicolumn{8}{|l|}{$\mathrm{S}_{\mathrm{pO}_{2}}, \%$} \\
\hline Initial & $94.0 \pm 2$ & $93.2 \pm 2$ & $0.0 \pm 0.0$ & $93.5 \pm 2.2$ & $93.6 \pm 2.5$ & $0.0 \pm 0.0$ & $0.0(0.0-0.0)$ \\
\hline Final & $90.6 \pm 3.7$ & $89.3 \pm 6.3$ & $0.0 \pm 0.0$ & $87.8 \pm 4.5$ & $88.0 \pm 4.4$ & $0.0 \pm 0.0$ & $0.0(0.0-0.0)$ \\
\hline \multicolumn{8}{|c|}{ 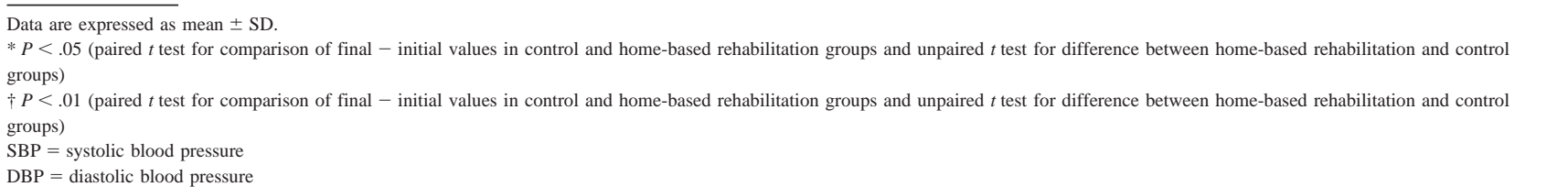 } \\
\hline
\end{tabular}

Table 4. SGRQ Scores Before and After Evaluations in Control and Home-Based Rehabilitation Groups

\begin{tabular}{|c|c|c|c|c|c|c|c|}
\hline & \multicolumn{3}{|c|}{ Control Group $(n=15)$} & \multicolumn{4}{|c|}{ Home-Based Rehabilitation Group $(n=29)$} \\
\hline & Initial & Final & $\begin{array}{c}\text { Difference } \\
\text { (Final - Initial) }\end{array}$ & Initial & Final & $\begin{array}{c}\text { Difference } \\
\text { (Final - Initial) }\end{array}$ & $\begin{array}{c}\text { Home-Based } \\
\text { Rehabilitation vs Control } \\
(95 \% \mathrm{CI})\end{array}$ \\
\hline Symptoms & $50.8 \pm 18.9$ & $47.7 \pm 22.1$ & $-3.1 \pm 21.0$ & $57.0 \pm 19.4$ & $47.9 \pm 18.9$ & $-9.1 \pm 21.0$ & $6.0(-19.4$ to 7.4$)$ \\
\hline Activities & $63.1 \pm 23.1$ & $66.3 \pm 24.9$ & $3.2 \pm 15.7$ & $54.2 \pm 25.3$ & $46.1 \pm 25.1$ & $-8.1 \pm 24.1$ & $11.4(-25.3$ to 2.5$)$ \\
\hline Impact & $43.8 \pm 29.3$ & $48.5 \pm 30.2$ & $4.7 \pm 12.3$ & $47.3 \pm 24.8$ & $43.3 \pm 23.2$ & $-4.0 \pm 20.6$ & $8.7(-20.4$ to 3.0$)$ \\
\hline Total & $49.1 \pm 23.2$ & $52.3 \pm 24.5$ & $3.1 \pm 12.2$ & $50.3 \pm 20.9$ & $43.6 \pm 18.5$ & $-6.4 \pm 16.1^{*}$ & $9.7(-1.0 \text { to }-0.1)^{*}$ \\
\hline
\end{tabular}




\section{Home-Based Pulmonary Rehab for Subjects With COPD}

monary rehabilitation protocol for patients with COPD, and it demonstrates functional benefits.

\section{Conclusions}

This study demonstrates that a simple, low-cost, homebased pulmonary rehabilitation program adapted to reallife situations leads to improvements in exercise capacity, shortness of breath, and quality of life. The findings underscore the importance of home-based pulmonary rehabilitation as a treatment option for patients with COPD, reaching a greater number of patients. Further studies should be carried out to evaluate long-term adherence to this type of program.

\section{REFERENCES}

1. Rabe KF, Hurd S, Anzueto A, Barnes PJ, Buist SA, Calverley P, et al. Global strategy for the diagnosis, management, and prevention of chronic obstructive pulmonary disease: GOLD executive summary. Am J Respir Crit Care Med 2007;176(6):532-555.

2. Nici L, Donner C, Wouters E, Zuwallack R, Ambrosino NBJ. American Thoracic Society/European Respiratory Society statement on pulmonary rehabilitation. Am J Respir Crit Care Med 2006;173(12): 1390-1413.

3. Ries AL, Bauldoff GS, Carlin BW, Casaburi R, Emery CF, Mahler DA, et al. Pulmonary rehabilitation: joint ACCP/AACVPR evidencebased clinical practice guidelines. Chest 2007;131(5 Suppl):4S-42S.

4. Wijkstra PJ, Ten Vergert EM, van Altena R, Otten V, Kraan J, Postma DS, Koëter GH. Long term benefits of rehabilitation at home on quality of life and exercise tolerance in patients with chronic obstructive pulmonary disease. Thorax 1995;50(8):824-828.

5. Murphy N, Bell C, Costello RW. Extending a home from hospital care programme for COPD exacerbations to include pulmonary rehabilitation. Respir Med 2005;99(10):1297-1302.

6. Behnke M, Taube C, Kirsten D, Lehnigk B, Jörres RA, Magnussen $\mathrm{H}$. Home-based exercise is capable of preserving hospital-based improvements in severe chronic obstructive pulmonary disease. Respir Med 2000;94(12):1184-1191.

7. Singh V, Khandelwal DC, Khandelwal R, Abusaria S. Pulmonary rehabilitation in patients with chronic obstructive pulmonary disease. Indian J Chest Dis Allied Sci 2003;45(1):13-17.

8. Boxall AM, Barclay L, Sayers A, Caplan GA. Managing chronic obstructive pulmonary disease in the community. A randomized controlled trial of home-based pulmonary rehabilitation for elderly housebound patients. J Cardiopulm Rehabil 2005;25(6):378-385.
9. Maltais F, Bourbeau J, Shapiro S, Lacasse Y, Perrault H, Baltzan M, et al. Effects of home-based pulmonary rehabilitation in patients with chronic obstructive pulmonary disease. Ann Intern Med 2008; 149(12):869-878.

10. Miller MR, Hankinson J, Brusasco V, Burgos F, Casaburi R, Coates A, et al. Standardisation of spirometry. Eur Respir J 2005;26(2): 319-338.

11. Hankinson JL, Odencrantz JR, Fedan KB. Spirometric reference values from a sample of the general U.S. population. Am J Respir Crit Care Med 1999;159(1):179-187.

12. Sousa TC, Jardim JR, Jones PW. Validation of the Saint George respiratory questionnaire (SGRQ) in chronic obstructive pulmonary disease in Brazil. J Bras Pneumol 2000;26(3):119-128.

13. Committee on Proficiency Standards for Clinical Pulmonary Function Laboratories. ATS statement: guidelines for the six-minute walk test. Am J Respir Crit Care Med 2002;166(1):111-117.

14. Faul F, Erdfelder E, Buchner A, Lang AG. Statistical power analyses using G*Power 3.1: tests for correlation and regression analyses. Behav Res Methods 2009;41(4):1149-1160.

15. Fernández AM, Pascual J, Ferrando C, Arnal A, Vergara I, Sevila V. Home-based pulmonary rehabilitation in very severe COPD: is it safe and useful? J Cardiopulm Rehabil Prev 2009;29(5):325-331.

16. Holland AE, Hill CJ, Rasekaba T, Lee A, Naughton MT, McDonald CF. Updating the minimal important difference for six-minute walk distance in patients with chronic obstructive pulmonary disease. Arch Phys Med Rehabil 2010;91(2):221-225.

17. Puhan MA, Chandra D, Mosenifar Z, Ries A, Make B, Hansel NN, et al. The minimal important difference of exercise tests in severe COPD. Eur Respir J 2011;37(4):784-790.

18. Porto EF, Castro AA, Nascimento O, Oliveira RC, Cardoso F, Jardim JR. Modulation of operational lung volumes with the use of salbutamol in COPD patients accomplishing upper limbs exercise tests. Respir Med 2009;103(2):251-257.

19. Osterberg L. Drug therapy: adherence to medication. N Engl J Med 2005;353(5):487-497.

20. Vermeire E, Hearnshaw H, Van Royen P, Denekens J. Patient adherence to treatment: three decades of research. J Clin Pharm Ther 2001;26(5):331-342.

21. Schneider C, Jick SS, Bothner U, Meier CR. COPD and the risk of depression. Chest 2010;137(2):341-347.

22. Jones PW. Health status measurement in chronic obstructive pulmonary disease. Thorax 2001;56(11):880-887.

23. Cambach W, Chadwick-Straver RV, Wagenaar RC, van Keimpema AR, Kemper HC. The effects of a community-based pulmonary rehabilitation programme on exercise tolerance and quality of life: a randomized controlled trial. Eur Respir J 1997;10(1):104-113.

24. O'Donnell DE, Lam M, Webb KA. Measurement of symptoms, lung hyperinflation, and endurance during exercise in chronic obstructive pulmonary disease. Am J Respir Crit Care Med 1998;158(5): 1557-1565. 